\title{
Hardware Architecture Design for Explicit Model Predictive Control
}

\author{
Tor A. Johansen ${ }^{1}$, Warren Jackson ${ }^{2}$, Robert Schrieber ${ }^{2}$, Petter Tøndel ${ }^{1}$ \\ ${ }^{1}$ Department of Engineering Cybernetics, \\ Norwegian University of Science and Technology, NO-7491 Trondheim, Norway. \\ ${ }^{2}$ Hewlett-Packard Laboratories, Palo Alto, California 94304-1126, USA
}

\begin{abstract}
Solutions to constrained linear and hybrid model predictive control (MPC) can be explicitly characterized in terms of piecewise linear (PWL) state feedback control. A PWL controller is pre-computed using parametric programming, and the exact explicit MPC implementation corresponds to the evaluation of a PWL function in the control unit. It has recently been shown that such evaluation can be boosted by binary search tree data structures. We report hardware architecture design results for this type of PWL control, and show that explicit MPC solutions can be implemented in a standard field programmable gate array (FPGA) or an application specific integrated circuit (ASIC) with about 20 kGates leading to computation times around one microsecond per evaluation for typical applications. This facilitates implementation of constrained MPC in small-scale industrial and consumer electronics applications that are characterized by fast sampling or low cost, such as mechatronics, MEMS, rotating machinery, power electronics, and acoustics.
\end{abstract}

\section{INTRODUCTION}

Model predictive control (MPC) is a powerful control method that allows constraints and MIMO systems to be treated systematically. Until recently, MPC has been exclusively used for relatively slow systems, mainly process plants. However, fast implementation of model predictive control based on online optimization in real-time systems has been considered in [1], [2], [3], [4], [5], [6], [7] for applications such as MEMS. Several modifications of the conventional MPC formulations that reduces online computational complexity has also been suggested [8], [9].

Alternatively, it has recently been shown that several classes of constrained MPC problems admit explicit piecewise linear (PWL) solutions. This includes constrained linear MPC [10], [11], [12], approximate explicit PWL solutions to nonlinear constrained MPC [13], [14], and hybrid MPC [15]. Implementation of explicit MPC amounts to the relatively simple task of evaluating a pre-computed PWL function.

A binary search tree representation of arbitrary polyhedral PWL functions was suggested in [16], [17]. It leads to very low requirements for processing in the control unit, but requires additional memory to store a pre-computed binary search tree data structure. In this work we report some results on hardware implementation of PWL function evaluation based on such a data structure.
Compared to conventional MPC, that relies on extensive real-time numerical optimization, the benefits include verifiability, low computational complexity, no need for floating point arithmetics (no recursive numerical computations), and deterministic execution. The main limitation of the explicit MPC approach is that the offline computational load (during synthesis) and control unit memory requirements usually increase quickly with the dimension and complexity of the problem, making it useful mainly for small scale problems. Still, this may not be prohibitive in many application areas. The main contribution of the present paper is to consider the option of direct hardware implementation using a high-level hardware synthesis tool [18], which may prove to be beneficial or cost-efficient in some applications.

\section{Explicit Model Predictive Control}

Below we give a short summary of linear MPC problems and their explicit solutions. Consider the linear system

$$
\begin{aligned}
x_{t+1} & =A x_{t}+B u_{t} \\
y_{t} & =C x_{t}
\end{aligned}
$$

where $x_{t} \in \mathbb{R}^{n}$ is the state variable, $u_{t} \in \mathbb{R}^{m}$ is the input variable, $A \in \mathbb{R}^{n \times n}, B \in \mathbb{R}^{n \times m}$ and $(A, B)$ is a controllable pair. The output and the control input are subject to the bounds

$$
y_{\min } \leq y_{t} \leq y_{\max }, \quad u_{\min } \leq u_{t} \leq u_{\max }
$$

where $y_{\min }<y_{\max }$ and $u_{\min }<u_{\max }$. For the current $x_{t}$, MPC solves the optimization problem

$$
\begin{aligned}
J^{*}\left(x_{t}\right)= & \min _{u_{t}, \ldots, u_{t+M-1}}\left\|x_{t+N \mid t}\right\|_{p}^{P} \\
& +\sum_{k=0}^{N-1}\left(\left\|x_{t+k+1 \mid t}\right\|_{p}^{Q}+\left\|u_{t+k}\right\|_{p}^{R}\right)
\end{aligned}
$$

subject to

$$
\begin{aligned}
& y_{\min } \leq y_{t+k \mid t} \leq y_{\max }, k=1, \ldots, N \\
& u_{\min } \leq u_{t+k} \leq u_{\max }, k=0, \ldots, N-1 \\
& x_{t \mid t}=x_{t} \\
& x_{t+k+1 \mid t}=A x_{t+k \mid t}+B u_{t+k}, k \geq 0 \\
& y_{t+k \mid t}=C x_{t+k \mid t}, k \geq 0 \\
& u_{t+M+k}=0 \quad \forall k \geq 0
\end{aligned}
$$


where $x_{t+k \mid t}$ is a $k$-step prediction of $x_{t+k}$ from the initial state $x_{t}$. Additionally we may require the terminal constraint

$$
L x_{t+N} \leq l
$$

to be satisfied. For $p=2,\|x\|_{p}^{E}=x^{T} E x^{1}, Q=Q^{T} \geq$ $0, R=R^{T}>0$ and $P \geq 0$. For $p=1$ and $p=\infty$, $\|x\|_{p}^{E}=\|E x\|_{p}$. For ease of notation, we may in the sequel skip the index $t$, and use $u$ for $u_{t}$ and $x$ for $x_{t}$. These problems can be reformulated and solved as the following multi-parametric programs:

1) $\mathrm{mp}-\mathrm{QP}(p=2)$ :

$$
\begin{aligned}
& \min _{U} U^{T} H U+x^{T} F U \\
& \text { s.t. } G U \leq W+S x,
\end{aligned}
$$

where $U=\left[u_{t}^{T}, \ldots u_{t+M-1}^{T}\right]^{T}$, see [11] for details. 2) $\operatorname{mp}-\mathrm{LP}(p=1$ or $p=\infty)$ :

$$
\begin{array}{r}
\min _{U} h^{T} U \\
\text { s.t. } G U \leq W+S x,
\end{array}
$$

where $U=\left[u_{t}^{T}, \ldots, u_{t+M-1}^{T} \epsilon^{T}\right]^{T}$ and $\epsilon$ is a vector of slack variables, see [19] for details.

3) Robust MPC $(p=\infty)$. In [20] the authors show that when introducing uncertainty to the linear model (1), that is

$$
x_{t+1}=A(w(t)) x_{t}+B(w(t)) u_{t}+E v(t),
$$

where $v(t)$ and $w(t)$ are unknown input disturbances and parametric uncertainties, respectively, a min-max optimization problem analogous to (3)-(9) can be solved by $N$ mp-LPs.

4) $\operatorname{mp}$-MILP $(p=1$ or $p=\infty)$ : Here the linear model (1) is replaced by the piecewise affine model.

$$
x_{t+1}=A_{i} x_{t}+B_{i} u_{t}+f_{i}, \text { if }\left[\begin{array}{l}
x_{t} \\
u_{t}
\end{array}\right] \in \mathcal{X}_{i},
$$

where $f_{i} \in \mathbb{R}^{n}$ are constant vectors and $\left\{\mathcal{X}_{i}\right\}$ is a polyhedral partition of the state+input space. The problem can be reformulated as a mp-MILP, see [21].

Similar formulations can also be given for similar and extended MPC problems, e.g. with non-zero setpoint, input rate constraints, infeasibility handling etc. [11].

These control design methods described above result in PWL functions $k: \mathbb{R}^{n} \rightarrow \mathbb{R}^{m}$ represented as

$$
k(x)=\left\{\begin{array}{cl}
K_{1} x+g_{1}, & \text { if } x \in X_{1} \\
K_{2} x+g_{2}, & \text { if } x \in X_{2} \\
\vdots & \\
K_{N} x+g_{N}, & \text { if } x \in X_{N}
\end{array}\right.
$$

with a polyhedral partition $\mathcal{P}=\left\{X_{1}, \ldots . ., X_{N}\right\}$ where the polyhedral sets are represented by linear inequalities (hyperplanes)

$$
X_{i}=\left\{x \in \mathbb{R}^{n} \mid A_{i} x \leq b_{i}\right\}
$$

\footnotetext{
${ }^{1}$ Although this does not strictly define a norm, we choose this description for ease of notation.
}

for $i=1, \ldots, N$. Such a partition satisfies $\operatorname{int} X_{i} \cap \operatorname{int} X_{j}=\emptyset$ for $i \neq j$ (they intersect only at the boundary), where int $X_{i}$ denotes the open interior of the set $X_{i}$. The PWL controller is completely characterized by the following matrices and vectors: $\left\{K_{i}, g_{i}, A_{i}, b_{i}\right\}_{i=1}^{N}$.

The controller output will be given by the PWL function $u_{t}=k\left(x_{t}\right)$ and the argument $x_{t}$ will typically change at every sampling instant. Controller implementation thus requires evaluation of a PWL function (17)-(18) at each sampling instant in the control unit.

In some variations of explicit MPC, such as [22], the polyhedral sets $X_{i}$ are represented by vertices

$$
X_{i}=\operatorname{conv}\left(v_{i}^{1}, v_{i}^{2}, \ldots, v_{i}^{L}\right)
$$

where $\operatorname{conv}()$ denotes the convex hull. These representations are equivalent, but require some modification of the algorithms used for evaluation. In other variants of explicit MPC, such as [14], [23], the partition has an orthogonal structure (quad-tree or $k-d$-tree) that further reduces computational complexity since the partition consists of hyper-rectangles

$$
X_{i}=\left\{x \in \mathbb{R}^{n} \mid b_{i}^{\ell} \leq x \leq b_{i}^{u}\right\}
$$

rather than general polyhedra.

\section{POLYHEDRAL PARTITION SEARCH STRATEGIES}

A typical polyhedral partition used to define a PWL function solving an MPC problems contains several hundred or thousands of polyhedra, even if complexity reduction methods such as [24], [25] are used. Often, neighboring regions contain the same linear mapping, cf. (17), such that the number of linear mapping coefficient matrices to store is significantly less than the number of polyhedral regions that must be stored as linear inequality coefficient matrices.

\section{A. Binary search tree representation}

Evaluating a PWL function (17) for a given $x$ consists of two steps:

- First, identify the polyhedral region index $i$ such that $x \in X_{i}$, and

- second, evaluate the linear function $K_{i} x+g_{i}$.

The second step is completely straightforward, while the first step can be implemented using a computationally efficient strategy described in [17]. It builds a binary search tree data structure that supports efficient search for the polyhedral region $X_{i}$ that satisfies $x \in X_{i}$. The overall idea is based on the observation that evaluation of a linear expression $c_{i} x-d_{i}$ corresponding to a single hyper-plane cut $c_{i} x-d_{i}=0$ may significantly reduce the number of candidate polyhedral regions. This is illustrated in Figure 1 , where the partition $\mathcal{P}=\left\{X_{1}, X_{2}, X_{3}, X_{4}, X_{5}, X_{6}, X_{7}\right\}$ contains only 7 polyhedra. Consider the point $x$ near the center of the area. In order to determine which polyhedron this point belongs to, the linear expression $c_{1} x-d_{1}$ may be evaluated. This hyper-plane essentially cuts the partition into two parts: $\left\{X_{1}, X_{2}, X_{3}\right\}$ (below the hyper-plan) and $\left\{X_{4}, X_{5}, X_{6}, X_{7}\right\}$ (above the hyper-plane). With the given 


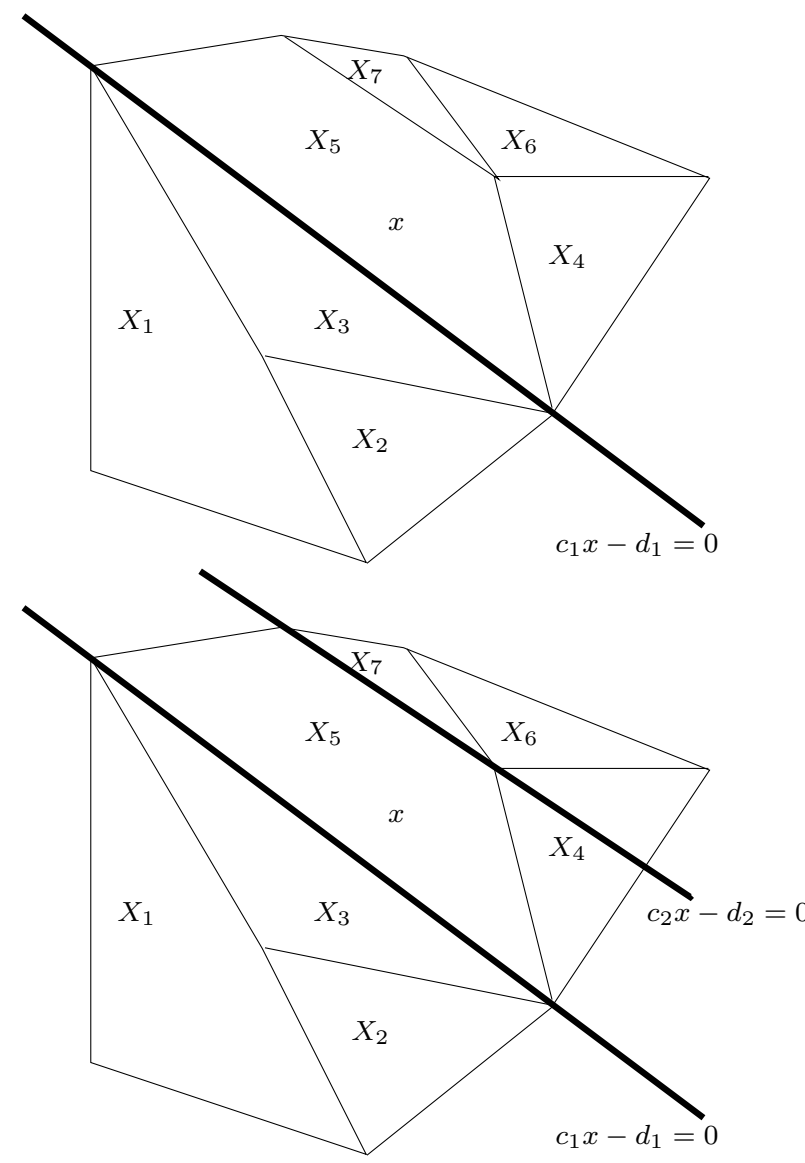

Fig. 1. Hyper-plane cuts of polyhedral partition.

$x$, the sign of the evaluated expression $c_{i} x-d_{i}$ shows that $x$ is located above the hyper-plane, and one can infer that $x \in X_{4} \cup X_{5} \cup X_{6} \cup X_{7}$. In other words, 3 out of 7 polyhedral regions has been excluded, and the remaining problem is greatly reduced. This procedure can be repeated, as shown in the 2nd part of Figure 1. By evaluating the linear expression $c_{2} x-d_{2}$ one can infer that $x \in X_{4} \cup X_{5}$. In other words, 2 out of 4 remaining regions have been eliminated and the problem is again greatly reduced. Since $X_{4}$ and $X_{5}$ are separated by a single hyper-plan, one can easily detect that $x \in X_{5}$ by evaluating a third linear expression. In summary, evaluation of three linear expressions is sufficient to determine which polyhedral region $x$ belongs to in this case. We observe that each region is characterized by 34 linear inequalities, such that an exhaustive search may require the evaluation of more than 20 linear expressions in this case. For general algorithms to construct such a binary search tree, we refer to [17].

The procedure illustrated above is completely general, and corresponds to the construction and traversal of a binary search tree where at each node there is a linear expression corresponding to a hyper-plane that cuts the remaining partition into three parts: Polyhedra that are completely on one side of the hyper-plane, polyhedra that are completely on the other side of the hyper-plane, and polyhedra that are cut by the hyper-plane. Estimating that each of these parts are of similar size, each node in the search tree will exclude approximately $1 / 3$ of the polyhedral regions. This leads to a search tree depth and computational complexity that is logarithmic in the number of polyhedra in the partition [17].

\section{B. Numerical round-off errors}

Due to the complicated numerical computations that leads to the polyhedral representation of the PWL function, see the references in the introduction, the mathematical partition property int $X_{i} \cap \operatorname{int} X_{j}=\emptyset$ for $i \neq j$ and $X_{1} \cup X_{2} \cup \ldots \cup$ $X_{N}=\bar{X}(\bar{X}$ is the whole region of interest $)$ will hold only "approximately". This means that some regions may slightly overlap, and there may be small "numerical gaps" between some regions. The binary search procedure will automatically complete the partition since when a leaf node is reached, the corresponding linear function will be evaluated without regard to numerical errors in the representation of the polyhedra. This means that the linear functions will be extended to cover the small "numerical gaps". Likewise, the boundary linear functions will be extrapolated outside the original partition if $x$ happens to be located outside the partition.

The insensitivity to numerical errors and lack of recursive numerical computations means that fixed point arithmetics may be utilized without any complications since round-off errors cannot accumulate. The accuracy of implementation will decrease gracefully as the number of bits used to represent the numerical data decreases (roundoff errors).

\section{Binary tree search algorithm}

The PWL function evaluation problem consists of executing at each sample two sets of nested loops

- Tree search loop: Starting at the root node of the binary search tree the loop iterates through the nodes until a leaf node is reached. The leaf node identifies the linear expression to be evaluated to compute the PWL function value.

- Control evaluation loop: Evaluation of the linear expression $k(x)=K_{i} x+g_{i}$ where the index $i$ corresponds to the leaf node. This is a nested loop corresponding to a matrix multiplication operation.

Together the two nested loops form a control block. The total time for execution of a control block is the sum of the times for the two parts.

\section{HARDWARE DESIGN RESUlTS}

In this section we consider how the binary tree search algorithm in section III-C can be implemented in hardware, and how the complexity of the resulting hardware design scales with the problem parameters. The key parameters characterizing the PWL function complexity are defined in Table I. 


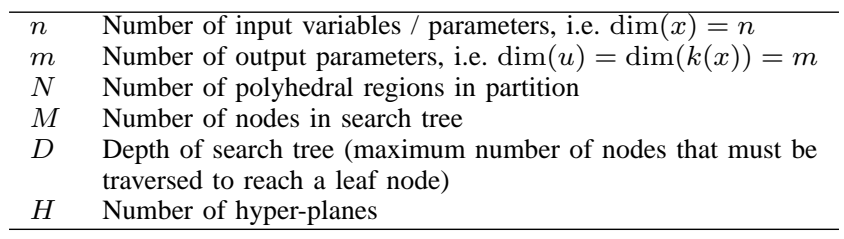

TABLE I

KEY PARAMETERS OF PWL FUNCTION EVALUATION PROBLEM.

\section{A. Hardware design approach}

The hardware design program PICO-Express (a product of Synfora Inc., http://synfora.com), based on the HP Labs PICO (Program In Chip Out) research [18] takes the C source code of PWL function evaluation, as described in section III-C, as an input along with some assumptions about the memory bandwidth. This program then computes an optimal architecture including cache size, functional register assignments, ALUs, etc. It also generates estimates of performance, execution time, memory requirements etc. PICO produces the hardware design expressed at the register-transfer level (RTL) in a hardware design language, either VHDL or Verilog. The RTL design is tested and verified by PICO as well. This design can be implemented in an FPGA or an ASIC. In either case, routing and placement tools, as well as libraries of parameterized macrocells (for the RTL level components such as adders, registers, etc.) are used to generate the FPGA or ASIC implementation.

The tree search and control evaluation loops are processed in a pipeline. It consists of a pipe-fill phase, the prolog, where iterations are initiated at regular intervals. These intervals are know as initiation intervals (II clock cycles). No iterations are complete during the prolog. Next, the loop enters the steady-state where every II cycles one iteration is completed and one new iteration is initiated. When the loop nears termination, it enters the pipe-drain state, know as the epilog, in which the pipeline is drained by allowing iterations to complete without new iterations being initiated. Iterations complete every II clock cycles until all iterations have completed.

The hardware design program tries to deliver a given initiation interval with a minimum gate count. The initiation interval is the number of clock cycles between sequential starts of the inner loop. An II of one can be achieved by PICO, since the inner loop recurrence is through an addition, and PICO can generate designs using a one-cycle adder. There is also an important dependence of larger latency. At the end of the inner loop, the dot product of the input/parameter vector $x$ with one hyperplane normal $c_{i}$ is first compared with a constant $d_{i}$, then a branch is taken to select one of two possible child nodes in the search tree, then the index of the selected node is used to start the lookup of the parameters of the next hyperplane from memory. To accommodate the latency of these operations and still achieve an II of one, the inner loop is padded with a few "slack" iterations at the front. These do nothing, but they increase the number of inner-loop iterations between

\begin{tabular}{lcccccc}
\hline Problem & $n$ & $m$ & $N$ & $M$ & $D$ & $H$ \\
\hline Double integrator $h=1$ & 2 & 1 & 5 & 13 & 4 & 10 \\
Double integrator $h=5$ & 2 & 1 & 25 & 87 & 7 & 86 \\
Double integrator $h=10$ & 2 & 1 & 95 & 323 & 9 & 346 \\
Double integrator $h=15$ & 2 & 1 & 215 & 815 & 11 & 818 \\
Quadruple integrator $h=2$ & 4 & 1 & 3 & 5 & 2 & 14 \\
Quadruple integrator $h=6$ & 4 & 1 & 31 & 215 & 8 & 268 \\
Quadruple integrator $h=10$ & 4 & 1 & 81 & 715 & 11 & 1188 \\
Heli $h=1$ & 6 & 2 & 47 & 527 & 11 & 82 \\
Heli $h=2$ & 6 & 2 & 152 & 15395 & 19 & 1107 \\
\hline
\end{tabular}

TABLE II

BENCHMARK PROBLEM CHARACTERISTICS, WHERE $h$ IS THE MPC HORIZON.

\begin{tabular}{lccc}
\hline Problem & $\begin{array}{c}\text { Gates } \\
\text { (kiloGates) }\end{array}$ & Clock cycles & $\begin{array}{c}\text { Loop time } \\
(\mu s)\end{array}$ \\
\hline Double integrator $h=1$ & 19.3 & 33 & 1.65 \\
Double integrator $h=5$ & 19.6 & 48 & 2.40 \\
Double integrator $h=10$ & 19.7 & 58 & 2.90 \\
Double integrator $h=15$ & 19.8 & 68 & 3.40 \\
Quadruple integrator $h=2$ & 19.7 & 31 & 1.55 \\
Quadruple integrator $h=6$ & 20.2 & 73 & 3.65 \\
Quadruple integrator $h=10$ & 20.4 & 94 & 4.70 \\
Heli $h=1$ & 21.7 & 129 & 6.45 \\
Heli $h=2$ & 22.9 & 201 & 10.5 \\
\hline
\end{tabular}

TABLE III

HARDWARE DESIGN RESULT SUMMARY WITH A CLOCK FREQUENCY ON 20 MHZ. THE TIMES WILL BE NEARLY A FACTOR OF 10 FASTER FOR A CLOCK FREQUENCY OF $200 \mathrm{MHz}$.

the completing one dot product and starting the next, so that there are enough cycles to cover this latency. For these experiments, all the tables of hyperplane normals and pointer arrays that define the search tree structure are assumed to be stored in fast SRAM in the accelerator device. PICO can also synthesize designs in which data arrays are kept in main memory.

\section{B. Benchmark problems}

The characteristics of the benchmark problems are given in Table II. The double integrator and helicopter examples are described in more detail in [26], and the quadruple integrator example in [27].

\section{Number of clock cycles per iteration}

The total time to execute one control block in clock cycles is summarized in Table III and illustrated in Figure 2. The number of clock cycles does not explicitly depend on the number of parameters but rather on the search depth of the tree. In fact the number of clock cycles, NClock is roughly given by

NClock $\approx D(n+1+\mathrm{SLACK})+m(n+1+\mathrm{SLACK})$

where SLACK is the number of padding iterations needed, as discussed above, and is 2 at $10-20 \mathrm{MHz}$ clock speeds and 4 for $200 \mathrm{MHz}$ clock speeds. This variable represents the time it takes to set up the inner loop and the number of clock cycles for memory access. The first term is the search time and the second is the function evaluation term. 

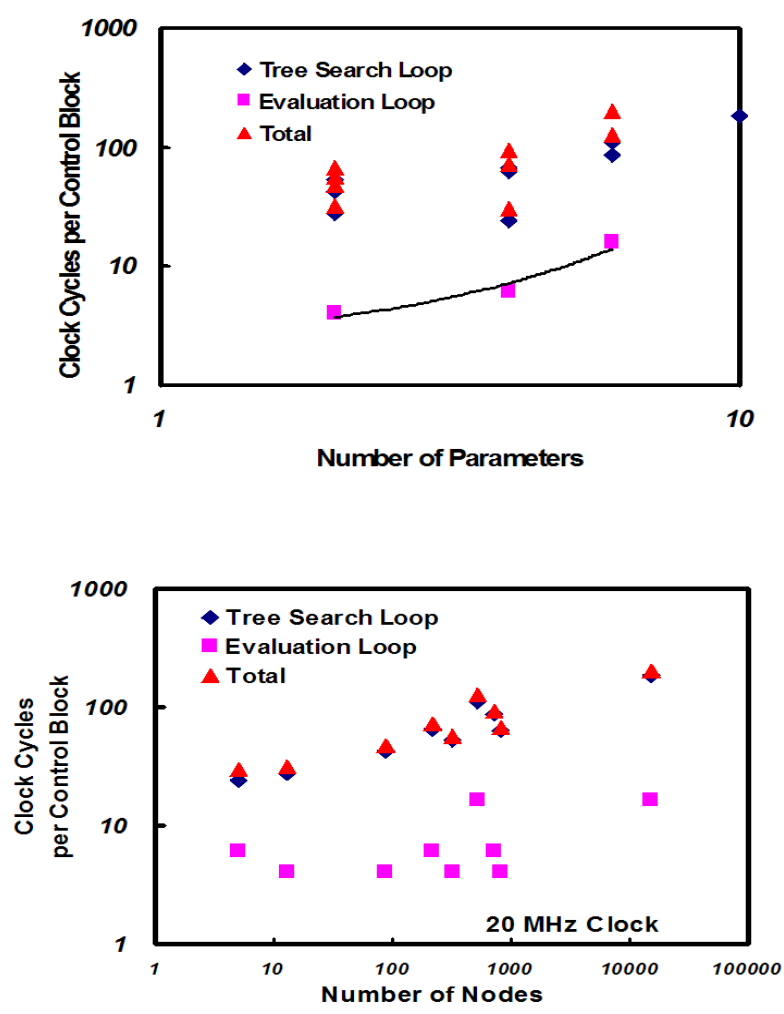

Fig. 2. The number of clock cycles to evaluate the PWL controller, as a function of the dimension $n$ of the parameters vector $x$ (upper) and as a function of the number of search tree nodes (lower).

Because the search depth increases as $\log _{2}(M)$, as expected, the execution time also increases as the $\log _{2}(M)$. This scaling can continue until the number of nodes is so large that the data can no longer be held in scratch SRAM memory and must be added as general system memory. Then the access time jumps to roughly 50 nsec. For clock speeds of 10 and $20 \mathrm{MHz}$, this is not a problem but for $200 \mathrm{MHz}$ clock speeds, this transition can lead to slower performance requiring a larger value for SLACK. In Figure 3 the dependence of the tree search clock cycles as a function of clock speed and number of nodes is shown. The dependence of the tree search on clock speed is not strong up to $200 \mathrm{MHz}$ indicating that for these problems clock speed translates directly into improved control loop speed. It should be mentioned that for $200 \mathrm{MHz}$ clock speeds, even the slowest benchmark problem can execute in about $1.1 \mu \mathrm{s}$.

\section{Number of gates}

The number of gates as a function of problem dimensions are given in Table III and Figure 4. Basically, the number of gates (20 kGates) for the tree search does not depend strongly on the problem parameters. The number of gates for the function evaluation also does not increase much with problem complexity. This result occurs because, in all cases, we have fixed the performance at one loop iteration

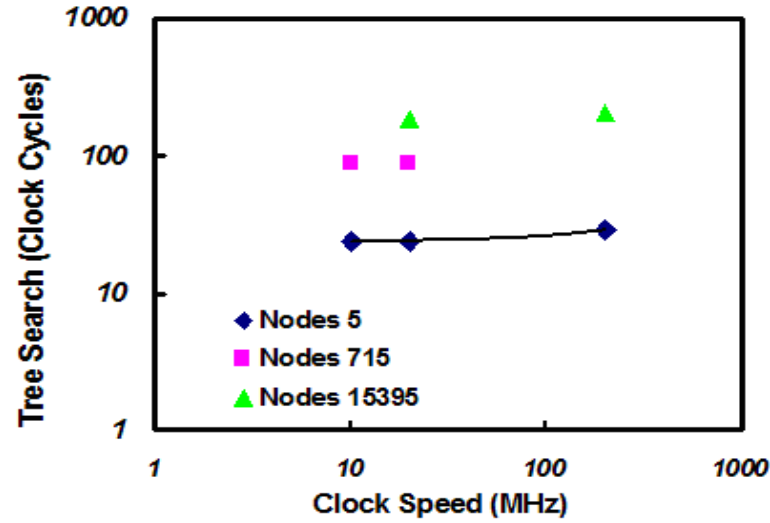

Fig. 3. Number of clock cycles to evaluate PWL controller, as a function of the chip clock speed. There is little overhead for increasing the clock speed up to $200 \mathrm{MHz}$. Higher speeds incur a significant penalty for memory access.

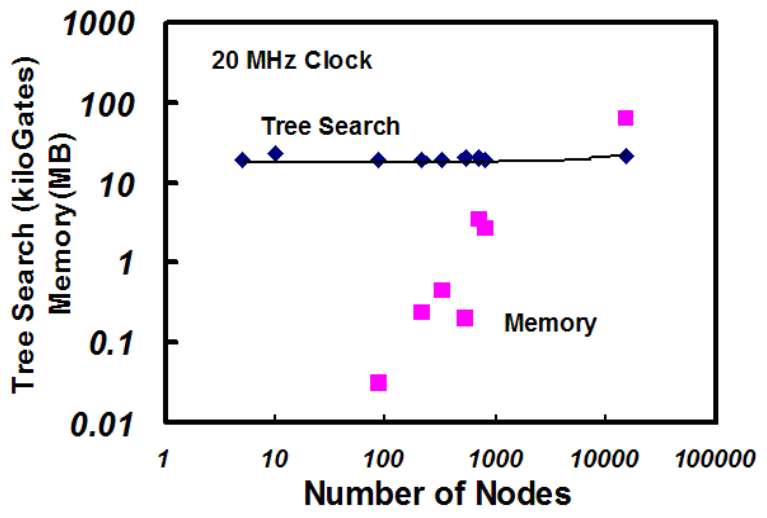

Fig. 4. The number of gates (diamonds) for the tree search and memory (squares) for the benchmark problems.

per clock cycle, and PICO has produced the least costly hardware that it can, while achieving this fixed (independent of parameters) throughput. Total latency, as indicated above, depends strongly on the parameters. If we were to change the performance requirement up or down, we would see an increase or a decrease in the gate count.

The size of the embedded SRAMs does not change, however, with performance, and these may dominate the chip cost. However, the number of SRAM cells scales directly with the number of search tree nodes, see Figure 4. For larger problems, this cost is by far the dominant cost of implementing the chip.

\section{Discussion ANd CONCLUSIONS}

If the clock frequency is assumed to be $200 \mathrm{MHz}$, then the control loop time would range from $0.2 \mu$ s to $1.1 \mu \mathrm{s}$ for the benchmark problems. This result is rather important in that it indicates that control for mechanical, thermal, and acoustic time scales can be handled by these controllers with speed to spare. In particular, lower level constrained multi-dimensional controls in mechanical systems such as 


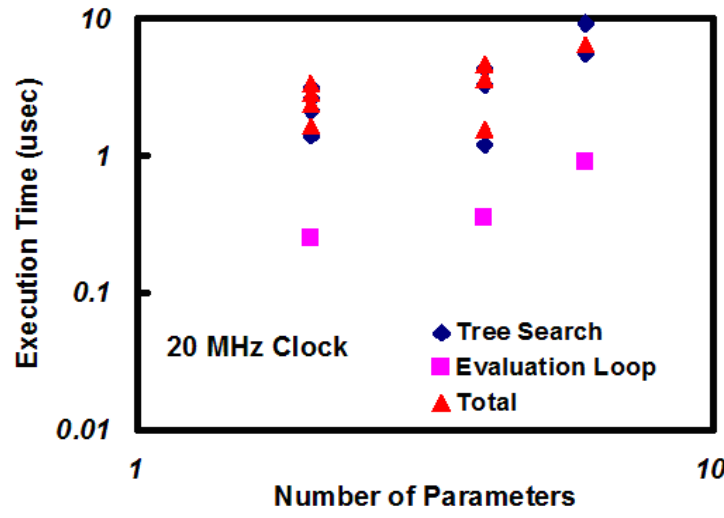

Fig. 5. Execution times for the various benchmark problems with a 20 $\mathrm{MHz}$ clock frequency. For $200 \mathrm{MHz}$ clock rates the times would be about 10 times faster.

robotics, cars etc. can readily be implemented using hardware implementation of explicit model predictive control. Because the complexity of the problem scales strongly with the number of parameters, this number should be minimized in order to improve the performance. The biggest cost factor appears to be the memory requirements for the number of nodes $M$, but reducing the complexity of the PWL function representation is in fact an active area of research [16], [26], [28], [24], [25], [23]. Moreover, a tradeoff between computational and memory requirements can be made by constructing a binary search tree of less depth such that at each leaf node a number of candidate linear expression can be compared using a sequential search [29].

In our case study, the hardware synthesis applied floating point arithmetics. Sufficient numeric accuracy will be achieved with implementation using fixed point arithmetics. This would lead to a significant reduction in memory requirements because data of lower resolution are stored, and in addition we may achieve a significant reduction in the number of gates.

\section{ACKNOWLEDGEMENTS}

This work was in part funded by the Research Council of Norway.

\section{REFERENCES}

[1] L. G. Bleris and M. V. Kothare, "Real-time implementation of model predictive control," in Proc. American Control Conference, Portland, OR, 2005, pp. 1752-1757.

[2] L. G. Bleris, J. Garcia, and M. V. Kothare, "Model predictive hydrodynamic regulation of microflows," in Proc. American Control Conference, Portland, OR, 2005, pp. 4166-4171.

[3] L. G. Bleris, M. V. Kothare, J. G. Garcia, and M. G. Arnold, "Towards embedded model predictive control for system-on-a-chip applications," J. Process Control, to appear, 2005.

[4] J. G. Garcia, M. G. Arnold, L. G. Bleris, and M. V. Kothare, "LNS architectures for embedded model predictive control processors," in Int. Conf. Compilers, Architectures and Synthesis for Embedded Systems, Washington DC, 2004, pp. 79-84.

[5] G. Hassapis, "Implementation of model predictive control using realtime multiprocessing computing," Microprocessors and Microsystems, vol. 27, pp. 327-340, 2003.
[6] D. Henrikson, A. Cervin, J. Akesson, and K. Arzen, "Feedback schedule of model predictive controllers," in Proc. 8th IEEE Realtime and embedded technology and applications symposium, San Jose, CA, 2002.

[7] W. Jackson, M. P. J. Fromherz, D. K. Biegelsen, J. Reisch, and D. Goldberg, "Constrained optimization based control of real time large-scale systems: Airjet object movement system," in Proc. IEEE Conf. Decision and Control, Orlando, 2001.

[8] B. Kouvaritakis, J. A. Rossiter, and J. Schuurmans, "Efficient robust predictive control," IEEE Trans. Automatic Control, vol. 45, pp. 1545-1549, 2000.

[9] M. Cannon and B. Kouvaritakis, "Efficient constrained model predictive control with asymptotic optimality," SIAM J. Optimization and Control, vol. 41, pp. 60-82, 2002.

[10] A. Bemporad, M. Morari, V. Dua, and E. N. Pistikopoulos, "The explicit solution of model predictive control via multiparametric quadratic programming," in Proc. American Control Conference, Chicago, 2000, pp. 872-876.

[11] A. Bemporad, M. Morari, V. Dua, and E. N. Pistikopoulos, "The explicit linear quadratic regulator for constrained systems," Automatica, vol. 38, pp. 3-20, 2002.

[12] P. Tøndel, T. A. Johansen, and A. Bemporad, "An algorithm for multiparametric quadratic programming and explicit MPC solutions," Automatica, vol. 39, pp. 489-497, 2003

[13] T. A. Johansen, "On multi-parametric nonlinear programming and explicit nonlinear model predictive control," in IEEE Conf. Decision and Control, Las Vegas, NV, 2002, vol. 3, pp. 2768-2773.

[14] T. A. Johansen, "Approximate explicit receding horizon control of constrained nonlinear systems," Automatica, vol. 40, pp. 293-300, 2004.

[15] A. Bemporad, F. Borrelli, and M. Morari, "Optimal controllers for hybrid systems: Stability and piecewise linear explicit form," in Proc. Conference on Decision and Control, Sydney, 2000.

[16] P. Tøndel, T. A. Johansen, and A. Bemporad, "Computation and approximation of piecewise affine control via binary search tree," in IEEE Conf. Decision and Control, Las Vegas, NV, 2002, vol. 3, pp. 3144-3149.

[17] P. Tøndel, T. A. Johansen, and A. Bemporad, "Evaluation of piecewise affine control via binary search tree," Automatica, vol. 39, pp. 945-950, 2003

[18] R. Schreiber, S. Aditya, S. Mahlke, V. Kathail, B. R. Rau, D. Cronquist, and M. Sivaraman, "PICO-NPA: High-level synthesis of nonprogrammable hardware accelerators," Journal of VLSI Signal Processing, vol. 31, pp. 127-142, June 2002.

[19] A. Bemporad, F. Borrelli, and M. Morari, "Model predictive control based on linear programming - the explicit solution," IEEE Trans. Automatic Control, vol. 47, pp. 1974-1985, 2002.

[20] A. Bemporad, F. Borrelli, and M. Morari, "Piecewise linear robust model predictive control," in Proc. European Control Conference, Porto, Portugal, Oct. 2001.

[21] A. Bemporad, F. Borrelli, and M. Morari, "Optimal controllers for hybrid systems: Stability and piecewise linear explicit form," in Proc. 39th IEEE Conf. on Decision and Control, Sydney, 2000.

[22] A. Bemporad and C. Filippi, "Approximate multiparametric convex programming," in Proc. 42th IEEE Conf. on Decision and Control, Maui, Hawaii, 2003, pp. 3185-3190.

[23] T. A. Johansen and A. Grancharova, "Approximate explicit model predictive control via orthogonal search tree," IEEE Trans. Automatic Control, vol. 48, pp. 810-815, 2003.

[24] T. Geyer, F.D. Torrisi, and M. Morari, "Optimal complexity reduction of piecewise affine models based on hyperplane arrangements," in American Control Conference, Boston, 2004, pp. 1190 - 1195.

[25] A. Bemporad, K. Fukuda, and F. D. Torrisi, "Convexity recognition of the union of polyhedra," Computational Gemometry, vol. 18, pp. $141-154,2001$.

[26] P. Tøndel and T. A. Johansen, "Complexity reduction in explicit model predictive control," in Preprints, IFAC World Congress, Barcelona, 2002.

[27] F. Borrelli, M. Baotic, A. Bemporad, and M. Morari, "Efficient online computation of explicit model predictive control," in Proc. IEEE Conf. Decision and Control, Orlando, 2001, vol. 2, pp. 1187-1192.

[28] P. Grieder, M. Kvasnica, M. Baotic, and M. Morari, "Low complexity control of piecewise affine systems with stability guarantee," in American Control Conference, Boston, 2004.

[29] T. A. Johansen, I. Petersen, and O. Slupphaug, "Explicit suboptimal linear quadratic regulation with input and state constraints," Automatica, vol. 38, pp. 1099-1112, 2002. 